\title{
An In Vitro Study on Prestin Analog Gene in the Bullfrog Hearing Organs
}

\author{
Zhongying Wang, ${ }^{1,2,3}$ Minfei Qian, ${ }^{1,2,3}$ Qixuan Wang, ${ }^{1,2,3}$ Huihui Liu, ${ }^{1,2,3}$ Hao Wu $\left(\mathbb{D},{ }^{1,2,3}\right.$ \\ and Zhiwu Huang ${ }^{1,2,3}$ \\ ${ }^{1}$ Department of Otolaryngology-Head and Neck Surgery, Shanghai Ninth People's Hospital, Shanghai Jiao Tong University School \\ of Medicine, Shanghai, China \\ ${ }^{2}$ Ear Institute, Shanghai Jiao Tong University School of Medicine, Shanghai, China \\ ${ }^{3}$ Shanghai Key Laboratory of Translational Medicine on Ear and Nose Diseases, Shanghai, China
}

Correspondence should be addressed to Hao Wu; haowu@sh-jei.org and Zhiwu Huang; huangzw86@126.com

Received 7 February 2020; Revised 22 April 2020; Accepted 20 May 2020; Published 2 July 2020

Academic Editor: Renjie Chai

Copyright (c) 2020 Zhongying Wang et al. This is an open access article distributed under the Creative Commons Attribution License, which permits unrestricted use, distribution, and reproduction in any medium, provided the original work is properly cited.

\begin{abstract}
The prestin-based active process in the mammalian outer hair cells (OHCs) is believed to play a crucial role in auditory signal amplification in the cochlea. Prestin belongs to an anion transporter family (SLC26A). It is densely expressed in the OHC lateral plasma membrane and functions as a voltage-dependent motor protein. Analog genes can be found in the genome of nonmammalian species, but their functions in hearing are poorly understood. In the present study, we used the gerbil prestin sequence as a template and identified an analog gene in the bullfrog genome. We expressed the gene in a stable cell line (HEK293T) and performed patch-clamp recording. We found that these cells exhibited prominent nonlinear capacitance (NLC), a widely accepted assay for prestin functioning as a motor protein. Upon close examination, the key parameters of this NLC are comparable to that conferred by the gerbil prestin, and nontransfected cells failed to display NLC. Lastly, we performed patch-clamp recording in HCs of all three hearing organs in bullfrog. HCs in both the sacculus and the amphibian papilla exhibited a capacitance profile that is similar to NLC while HCs in the basilar papilla showed no sign of NLC. Whether or not this NLC-like capacitance change is involved in auditory signal amplification certainly requires further examination; our results represent the first and necessary step in revealing possible roles of prestin in the active hearing processes found in many nonmammalian species.
\end{abstract}

\section{Introduction}

Hair cells (HCs) in the cochlea play a critical role in converting mechanical sound waves into neural signals for hearing [1-3]. The mammalian cochlea contains one row of inner hair cells (IHCs) that feed auditory signals to auditory afferent fibers, and three rows of outer hair cells (OHCs) that are able to contract upon depolarization and elongate when hyperpolarized [4-6]. This change of length (electromotility) happens at a microsecond time scale. This form of electromotility surprisingly does not require any force generator like ATP or calcium $[4,7,8]$. It is generally accepted that electromotility provides the physiological basis of a precise frequency selectivity and sensitivity of mammalian hearing $[5,9,10]$.
Electromotility is the result of conformational changes of a transmembrane protein named prestin. Prestin belongs to a highly versatile solute carrier 26 (SLC26A) in the anion transporter family [11-13]. Almost all the SLC26A members transport different anion substrates across epithelia, and the mammalian prestin is unique owing to its functions as a voltage-dependent motor protein [13, 14]. The voltage-dependent charge movement conferred by prestin's voltage sensor can be measured as a nonlinear capacitance (NLC) of the cell membrane. The NLC is often used as a substitute for direct measurements of the somatic motility in outer HCs and prestin-transfected cells because it is linked to cell motility and can be easily assayed experimentally $[5,13,15,16]$. 
Comparable to that in mammals, the inner ear of nonmammalian vertebrates varies significantly in anatomy across classes. Despite the fact that amphibian hair cells are not as highly differentiated as mammalian OHCs, their ears are also sensitive, sharply tuned, and can spontaneously emit sounds. Both spontaneous and evoked otoacoustic emissions from the American bullfrogs have been reported [17, 18]. The overall emission levels of amphibian ears are larger than those of avian and human ears $[19,20]$. The hair bundle and prestin motors in the avian auditory HCs together generate a force underlying amplification and frequency tuning [21, 22]. It remains unclear whether frog HCs have prestin and if frog prestin participates in the active process with the hair bundle.

The American bullfrog has been widely used as an animal model for the study of auditory physiology because of its well-developed middle and inner ear anatomy. The inner ear of the American bullfrog contains three auditory organs: the amphibian papilla (AP), the basilar papilla (BP), and the sacculus (S). The AP receives acoustic stimuli within a frequency range of $100 \mathrm{~Hz}-1250 \mathrm{~Hz}$, while the BP covers the higher portion of the auditory frequency range from about $1.2 \mathrm{kHz}$ to $4 \mathrm{kHz}$ [23]. The sacculus is a mixed-function organ which is most sensitive to low-frequency sounds $(120 \mathrm{~Hz} \pm 24 \mathrm{~Hz})$ and seismic sensation [24, 25]; however, none of these investigations have focused on prestin and electromotility. We generated stable cell lines transfected with the frog prestin by an AAVS1 site-specific integration. The NLC of the frog prestin, both in transfected cells and in primary $\mathrm{HCs}$ isolated from frog auditory organs, were measured using a patch-clamp technology. The goal of our work was to investigate whether frog HCs had prestin and if it functioned as an intrinsic motor for amplification and frequency selectivity with the hair bundle.

\section{Methods}

2.1. Cloning and Analyses of Prestin Orthologs. We obtained the prestin coding region of gerbil (Meriones unguiculatus), tropical clawed frog (Xenopus tropicalis), and the American bullfrog (Rana catesbeiana) using a BLAST analysis of the Ensembl and NCBI genomic databases. Genomic sequence data from gerbil and bullfrog were used to deduce the full coding cDNAs, which were then synthesized (HuaGene, China). The correct orientation and reading frame were verified by sequence analysis, and ortholog and paralog comparisons were conducted using UniProt, CLUSTALW, and Espript 3. All constructs were verified by gene sequencing.

\subsection{Generation of Stable Cell Lines That Express fPres and gPres}

2.2.1. Construction of Vectors for AAVS1 Site-Specific Integration. The AAVS1 safe harbor locus site-specific integration used CRISPR/Cas9-mediated gene editing. The sgRNA (GGGCCACTAGGGACAGGAT) targeting the AAVS1 site was cloned into a lentiviral vector (pLentiCRISPR), which contained a SpCas9 expression cassette. A donor vector was generated by assembling PCR-amplified fragments by restriction digestion and ligation. The resulting vector contained two homology arms from HEK293T geno- mic DNA that flanked an overexpression cassette with a puromycin selection marker on the plasmid backbone (pTOPO-AAVS1-EF1). This donor vector was designed for the expression of fPres- and gPres-enhanced GFP (EGFP) fusion proteins driven by the CMV promoter.

2.2.2. Cell Culture. HEK293T cells were cultured in the Dulbecco's Modified Eagle's Medium (DMEM) (Invitrogen, Carlsbad, CA, USA) supplemented with $10 \%$ fetal bovine serum (FBS) (Invitrogen) at $37^{\circ} \mathrm{C}$ in $5 \% \mathrm{CO}_{2}$. Mycoplasma testing was performed regularly using PCR detection. Cells were transfected at 60\%-80\% confluence using the Lipofectamine 2000 DNA transfection reagent (Thermo Fisher Scientific), typically with $2 \mu \mathrm{g}$ plasmid(s) and $5 \mu \mathrm{L}$ of the transfection reagent in a 6-well culture dish.

2.2.3. Expression of fPres and gPres in HEK293T Cells. Cells were cotransfected with a mixture of plasmids for sgRNA/Cas9 and the donor (donor : sgRNA/Cas9 $=1.5 \mu \mathrm{g}: 0.5 \mu \mathrm{g}$ ). Then, $2 \mu \mathrm{g} / \mathrm{mL}$ puromycin was added into the culture medium $24 \mathrm{~h}$ after transfection and cell pools expressing prestin and EGFP were identified after puromycin screening for $7 \mathrm{~d}-10 \mathrm{~d}$.

2.3. Confocal Imaging. The cells from the stable cell line at passage six were cultured for $12 \mathrm{~h}$ before immunodetection. Cells were rinsed with phosphate-buffered saline (PBS) one time and fixed with $4 \%$ paraformaldehyde for $30 \mathrm{~min}$. Then, the cells were washed twice for $15 \mathrm{~min}$ each before they were permeabilized with PBT (PBS, $1 \%$ Triton X-100) and blocked with $1 \%$ bovine serum albumin (BSA) in PBS for $1 \mathrm{~h}$ at room temperature (RT). Confocal imaging was conducted with a laser scanning microscope (Leica Microsystems, Germany) using a $63 x$ oil immersion objective.

2.4. Animals. Adult American bullfrogs (Rana catesbeiana) were purchased from a local vendor. Two-week-old C57 mice were purchased from the SIPPR-BK Laboratory Animal Ltd. (Shanghai, China). The care and use of animals were conducted in accordance with the Guide for the Care and Use of Laboratory Animals (National Institutes of Health, USA) and approved by the University Committee of Laboratory Animals of Shanghai Jiao Tong University.

Bullfrogs were sedated in an ice bath for $20 \mathrm{~min}$ and then double-pithed and decapitated. Amphibian papillae, basilar papillae, and sacculi were dissected and recorded in an extracellular solution containing (in mM) $95 \mathrm{NaCl}, 1 \mathrm{KCl}, 1 \mathrm{MgCl}_{2}$, 20 TEA-Cl, $0.5 \mathrm{CaCl}_{2}, 2 \mathrm{CoCl}_{2}$, and $10 \mathrm{HEPES}$ at $\mathrm{pH} 7.30$ (240 mosmol/L). $\mathrm{NaOH}$ was used for $\mathrm{pH}$ adjustment.

Cochleae and the apical coil of the organ of Corti were acutely dissected from C57 mice and fixed to a recording chamber. The external solution contained (mM) $120 \mathrm{NaCl}$, 20 TEA-Cl, $2 \mathrm{CoCl}_{2}, 2 \mathrm{MgCl}_{2}, 10 \mathrm{HEPES}$, and 5 glucose at $\mathrm{pH}$ 7.3. $\mathrm{NaOH}$ was used for $\mathrm{pH}$ adjustment.

2.5. Electrophysiology. Recordings of bullfrog HCs were performed at $20^{\circ} \mathrm{C}$ within $3 \mathrm{~h}$ of dissection. Patch pipettes were pulled from thick-walled borosilicate glass (World Precision Instruments) using a Narishige puller (model PP-830) to resistances of $5 \mathrm{M} \Omega-8 \mathrm{M} \Omega$ and coated with dental wax. 
Internal solutions for the bullfrog $\mathrm{HCs}$ were composed of (in $\mathrm{mM}) 100 \mathrm{CsCl}, 10 \mathrm{EGTA}, 10 \mathrm{HEPES}$, and $1 \mathrm{MgCl}_{2}$ at $\mathrm{pH} 7.30$ (240 mosmol/L). CsOH was used for $\mathrm{pH}$ adjustment. Wholecell voltage-clamp recordings were performed with an EPC10/2 (HEKA Electronics) patch-clamp amplifier and Pulse software (HEKA). The HCs were held at $-80 \mathrm{mV}$. Offline analysis was performed mainly with the Igor Pro 5.0 software (WaveMetrics).

We recorded mouse $\mathrm{OHCs}$ at $20^{\circ} \mathrm{C}$ within $1.5 \mathrm{~h}$ of dissection. Patch pipettes were pulled from thick-walled borosilicate glass (World Precision Instruments) using a Narishige puller (model PP-830) to resistances of about $6 \mathrm{M} \Omega$ and then coated with dental wax. The internal solution consisted of (mM) $140 \mathrm{CsCl}, 2 \mathrm{MgCl}_{2}, 10 \mathrm{EGTA}$, and 10 HEPES at $\mathrm{pH}$ 7.3. $\mathrm{CsOH}$ was used for $\mathrm{pH}$ adjustment. The osmolarity was adjusted to $300 \mathrm{mosmol} / \mathrm{L}$.

HEK cells were detached with trypsin (Invitrogen) treatment before recordings were collected. The detached cells were then bathed in an extracellular solution containing (in mM) $120 \mathrm{NaCl}, 20$ TEA-Cl, $2 \mathrm{CoCl}_{2}, 2 \mathrm{MgCl}_{2}, 10 \mathrm{HEPES}$, and 5 glucose at pH 7.2. Osmolarity was adjusted to 300 mos$\mathrm{mol} / \mathrm{L}$ with glucose. Recording pipettes were pulled with resistances of $2.5 \mathrm{M} \Omega-5.0 \mathrm{M} \Omega$ and filled with internal solution (in $\mathrm{mM}$ ): $140 \mathrm{CsCl}, 2 \mathrm{MgCl}_{2}, 10$ EGTA, and 10 HEPES. NLC measurements were performed on cultured cells with a robust membrane-associated EGFP expression. After rupture, we selected the cells whose membrane resistance was over $300 \mathrm{M} \Omega$ and showed normal $\mathrm{Cm}$ and $\mathrm{Rm}$ values.

The sine $+\mathrm{DC}$ software lock-in function of Patchmaster was used to obtain the voltage-sensor displacement currents and capacitance; a voltage protocol was designed that included both ramp and sine stimulation ( $800 \mathrm{~Hz}$ with a $10 \mathrm{mV}$ amplitude). Sine waves were superimposed onto ramps from $-150 \mathrm{mV}$ to $100 \mathrm{mV}$ for a duration of $300 \mathrm{~ms}$. The NLC was fitted with the derivative of a Boltzmann function:

$$
C m=\frac{\mathrm{Q}_{\max } \alpha}{\exp \left[\alpha\left(V_{m}-V_{1 / 2}\right)\right]\left(1+\exp \left[-\alpha\left(V_{m}-V_{1 / 2}\right)\right]\right)^{2}}+C_{\operatorname{lin}}
$$

where $Q_{\max }$ is the maximum charge transfer, $V_{1 / 2}$ is the voltage at half-maximum charge transfer, $C_{\operatorname{lin}}$ is the residual linear membrane capacitance, and $\alpha$ is the slope factor describing the voltage dependence. $\alpha=z e / k T$, where $k$ is Boltzmann's constant, $T$ is the absolute temperature, $z$ is the valence of charge movement, and $e$ is the electron charge.

\section{Results}

3.1. fPres Confers NLC to HEK293T Cells. In order to obtain the prestin coding region of the American bullfrog, we used a BLAST analysis of the Ensembl and NCBI genomic databases. Using the CLUSTAL method, alignment of the mouse, gerbil, Xenopus, and Rana prestin protein sequences was conducted (Figure 1). This alignment revealed nearly $97 \%$ identity among mouse and gerbil, 35\% among gerbil and Rana, and 57\% among gerbil and Xenopus. Our alignment results were consistent with former comparative peptide sequence analyses of mammalian prestins that were much more conserved with only minor changes, while prestins were quite variable among vertebrate species like the bony fish, amphibians, and birds [26].

We examined the electrophysiological properties from HEK cells transfected with the fPres-EGFP protein fusions by a site-specific gene transfer at the human AAV site 1 (AAVS1) [27-30]. Transgene expression is influenced by the integration site and some random insertions or transient transfections which can interfere with genes or disturb their transcription, while site-specific integration can minimize variations between different cells and constructs [31, 32]. We chose the gerbil prestin as a positive control, while cells transfected only with the EGFP-vector were a negative control. Membrane expression of fPres and gPres was examined using confocal microscopy. Both the fPres- and gPrestransfected cells showed similar patterns of membrane expression (Figure 2(a)).

Voltage stimulus used for capacitance recordings consisted of a sine wave superimposed onto a voltage ramp. We measured the NLC from the OHCs (Figure 2(b)) and transfected cells. Figure 2(c) shows the currents of the fPres- and gPrestransfected cells and the OHCs. The fPres-transfected cells had an NLC (the red curve) similar to the bell-shaped curve conferred by the gerbil and mouse prestin (Figure 2(d); black and blue curves). We could not detect NLC in cells transfected only with the EGFP-vector $(n=12)$. An example of a flat response has been presented in Figure 2(e).

Using the first derivative of the Boltzmann function, four parameters $\left(Q_{\max }, C_{\text {lin }}, V_{1 / 2}\right.$, and $\left.z\right)$ from nonlinear curve fitting of the NLC were calculated. Since the HEK cells varied in size, which is corelated with the $C_{\text {lin }}$ value, we normalized the $Q_{\max }$ to the $C_{\text {lin }}$ to compare the magnitude of the charge movement measured from cells of different sizes. We measured the mouse OHCs as a control.

The NLC measurements were analyzed from 15 gPresand 16 fPres-transfected cells. The means and SEMs of the gPres were $Q_{\max }=0.27 \pm 0.04(\mathrm{fC}), Q_{\max } / C_{\operatorname{lin}}=16.9 \pm 2$ $(\mathrm{fC} / \mathrm{pF}), V_{1 / 2}=-68.3 \pm 4.4(\mathrm{mV})$, and $z=0.74 \pm 0.04$. The means and SEMs of the fPres were $Q_{\max }=0.18 \pm 0.02(\mathrm{fC})$, $Q_{\max } / C_{\text {lin }}=14.9 \pm 2.02(\mathrm{fC} / \mathrm{pF}), \quad V_{1 / 2}=-58.1 \pm 3.5(\mathrm{mV})$, and $z=0.72 \pm 0.03$. The means and SEMs of the OHCs were $Q_{\max } / C_{\operatorname{lin}}=136.4 \pm 5.98(\mathrm{fC} / \mathrm{pF}), \quad V_{1 / 2}=-71.5 \pm 3.6(\mathrm{mV})$, and $z=0.77 \pm 0.03$. The magnitude of gPres and fPres NLC was considerably less than that of the OHC (Figures 3(a) and $3(\mathrm{~b}) ; P<0.005$, Student's $t$-test). The charge density represented by the $Q_{\max } / C_{\text {lin }}$ was not significantly different between fPres- and gPres-expressing cells; however, the charge density of both transfected cell lines was significantly lower than that measured in OHCs. Another functional parameter of $V_{1 / 2}$ is worth noting (Figure 3(c)). We observed no significant differences in $V_{1 / 2}$ between the gPres- and fPres-transfected cells, or between transfected cells and OHCs. Moreover, there were also no significant difference in the $z$ value between gPres, fPres, and the OHC (Figure 3(d)). All the data are shown in Table 1.

3.2. NLC Measurements of Frog HCs. The frog inner ear contains three auditory organs: the amphibian papilla (AP), the 

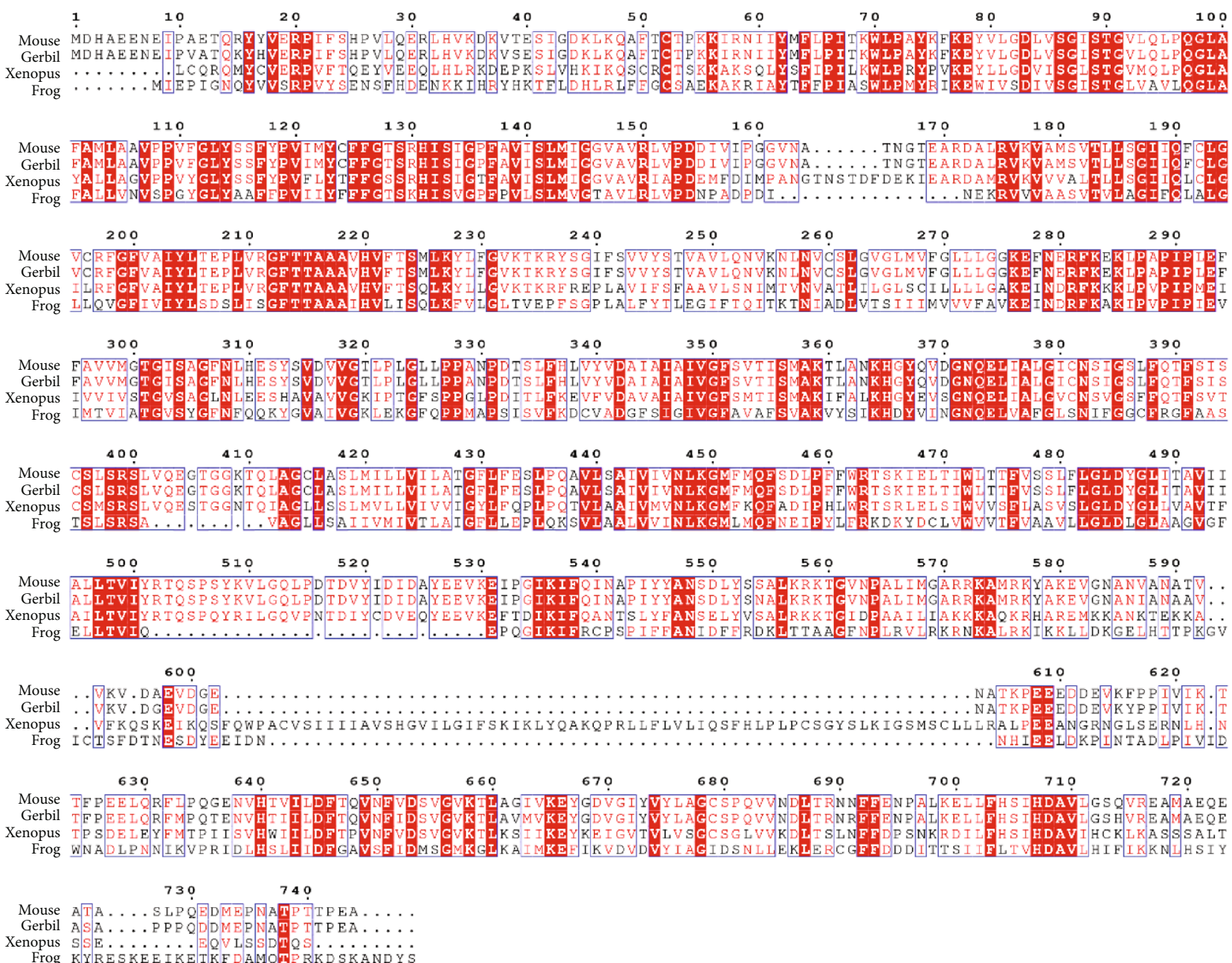

Figure 1: Alignment of amino acid sequences of SLC26A5 of mouse, gerbil, Xenopus, and bullfrog. Different colors had been used to represent identity of each residue among four species. Red block: full identity at a residue; red letter: partial identity at a residue; black: complete disparity at a residue. Gaps in the aligned sequences were indicated by the dashed line.

basilar papilla (BP), and the sacculus (S). The AP is composed of a patch of epithelium covered by HCs. The basilar papilla has a recess opening to the saccular space of the ear. The sacculus is a mixed-function organ which is sensitive to both hearing and vibration. The images of these three auditory organs are shown in Figures $4(\mathrm{a})-4(\mathrm{~g})$. Mammalian, avian, and lizard HCs are located on a basilar membrane. However, the frog inner ear lacks such a sensitive substrate for its sensory cells. Without the basilar membrane, the frog inner ear relies on the tectorial membrane and HCs for frequency selectivity [33].

We used the same voltage stimulus protocol to record the NLC of the HCs from the AP, BP, and S organs. All $\mathrm{AP}$ and S HCs displayed a bell-shaped voltage-dependent NLC (Figure 5(a)). Measurements were analyzed from 10 AP HCs and 8 S HCs (Figure 5(b)). The means and SEMs of the AP HCs were $Q_{\max }=10.4 \pm 1.4(\mathrm{fC}), Q_{\max } / C_{\text {lin }}=$ $14.9 \pm 1.01(\mathrm{fC} / \mathrm{pF}), V_{1 / 2}=-33.8 \pm 3.3(\mathrm{mV})$, and $z=1.8 \pm$ 0.16 . The means and SEMs of the S HCs were $Q_{\max }=19.9$ $\pm 2.4(\mathrm{fC}), Q_{\max } / C_{\operatorname{lin}}=16.4 \pm 0.68(\mathrm{fC} / \mathrm{pF}), \quad V_{1 / 2}=-20.7 \pm$ $3.3(\mathrm{mV})$, and $z=2.4 \pm 0.08$. The S HCs had a significant gain of NLC when compared to those from AP $(P<0.01)$. The NLC magnitude of AP and S HCs was significantly less than that of the OHCs $(P<0.005$, Student's $t$-test $)$, and the charge density of $S$ HCs was significantly higher than that of the AP HCs $(P<0.005$, Student's $t$-test $)$.

Compared with the mouse OHCs, the charge density of both the AP and $\mathrm{S}$ cells was significantly low. The $V_{1 / 2}$ of the AP HCs were more depolarized than that of the $S$ cells $(P<0.05)$, with a difference of approximately $10 \mathrm{mV}$. The $V_{1 / 2}$ of the OHCs shifted in an even more depolarized direction than that measured in frog cells $(P<0.005)$, with a difference in the $V_{1 / 2}$ between frog HCs and OHCs of about $45 \mathrm{mV}$. The $z$ value of the S HCs was significantly higher than that measured in AP cells $(P<0.01)$, while the $z$ values of both AP and S HCs were significantly higher than that of the OHCs $(P<0.005)$.

Notably, we did not observe bell-shaped curves in the BP HCs. As shown in Figure 5(a), the blue curve represents the BP NLC and no evident peak was observed with the voltage applied to AP and S cells; therefore, no fitting results were obtained from the BP cells. 

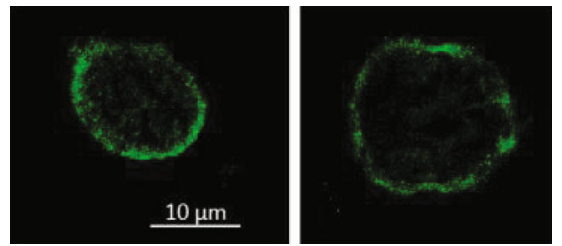

(a)

1.20

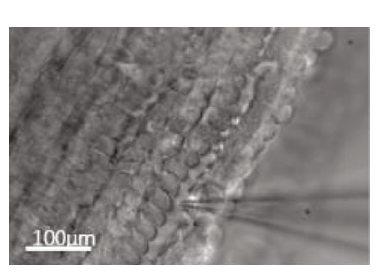

(b)

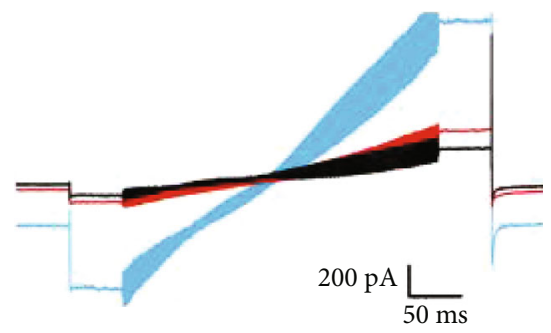

(c)
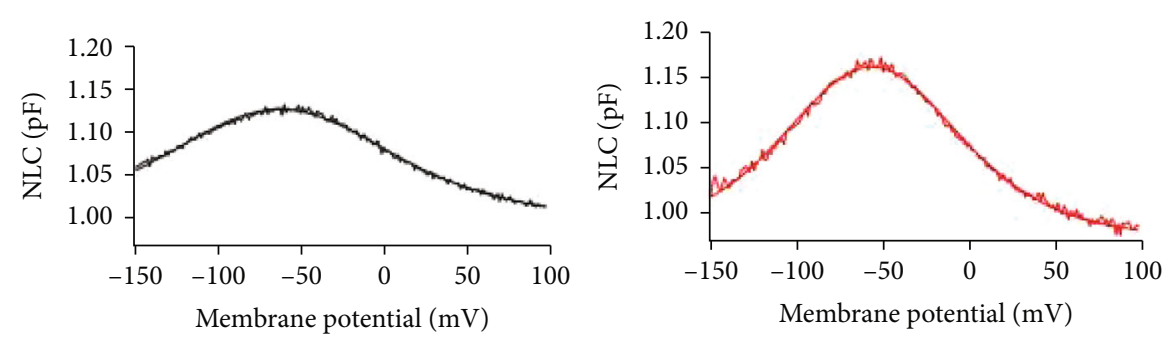

(d)

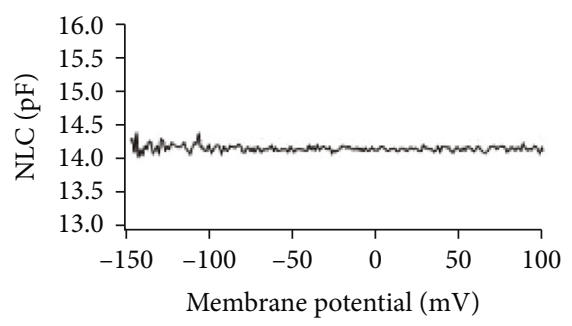

(e)

FIGURE 2: Nonlinear capacitance obtained from gPres- and fPres-transfected cells and a mouse OHC. (a) Confocal microscopy images of HEK cells transfected by gPres and fPres. (b) OHC patch. (c) Whole-cell currents of gPres- and fPres-transfected cells and OHC. Cells were held at $-80 \mathrm{mV}$ for current recordings. Voltage steps (300 ms in duration) varied from - 150 to $100 \mathrm{mV}$ in $10 \mathrm{mV}$ steps. Black-gPres, red-fPres, blueOHC. (d) NLC obtained from gPres- and fPres-transfected cells. Black-gPres, red-fPres. NLC obtained from the mouse OHC, blue curve. (e) This one showed the lack of detectable NLC in a representative control cell.

\section{Discussion}

Compared to mammals, many frog species do not have external ears or ear canals. In the frog family, a middle ear cavity is on the medial side of the tympanic membrane, which is coupled to the otic capsule via the stapes. The middle ear transmits acoustic information from the surrounding air to the inner ear, which contains fluid just like those of other vertebrates. Three distinct auditory organs are enveloped in this fluid-filled space: the amphibian papilla, the basilar papilla, and the sacculus. Low-frequency neurons that sense frequencies below $100 \mathrm{~Hz}$ innervate the sacculus, mid-frequency neurons that sense frequencies from 100 to $1000 \mathrm{~Hz}$ innervate the amphibian papilla, and highfrequency neurons that sense frequencies over $1000 \mathrm{~Hz}$ are connected to the basilar papilla [34].

The mammalian ear has frequency selectivity properties due to the propagation of an active traveling wave on the basilar membrane. In the mammalian inner ear, HCs are vulnerable to several forms of damage, including ototoxic drugs, inflammation, and aging [35-40]. The HCs play a critical role in converting mechanical sound waves into electrical signals along the pathway through the spiral gan- glion neurons to the cochlear nucleus [41]. The inner HCs serve as sensory receptors, and the outer HCs have the ability to improve cochlear sensitivity and frequency selectivity [10]. Together, they form the basilar membrane-OHC-tectorial membrane complex. What is unique to frogs is that no basilar membrane is attached to their auditory organs. There are no differentiated populations of $\mathrm{HCs}$ as there are in mammals. Although there are dramatic anatomical variations between mammals and amphibians, they continue to have many functional similarities. Like mammals, the frog inner ear has a sharp frequency selectivity and can generate both evoked and spontaneous otoacoustic emissions [17]. Since the mechanism found in mammalian ears does not develop in frog ears, additional mechanisms must contribute to the active process of nonmammalian auditory organs.

In order to investigate whether frog prestin was functional, we expressed fPres in HEK293T cells by sitespecific gene transferring at the human AAV site 1 . Our data showed that fPres produced robust NLC and responded to changes in the membrane potential just like its mammalian ortholog. We used cells transfected with gPres and EGFP alone as positive and negative controls, respectively, to test 


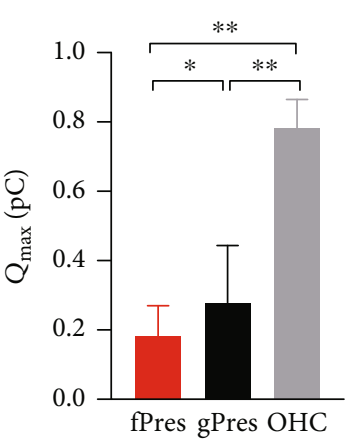

(a)

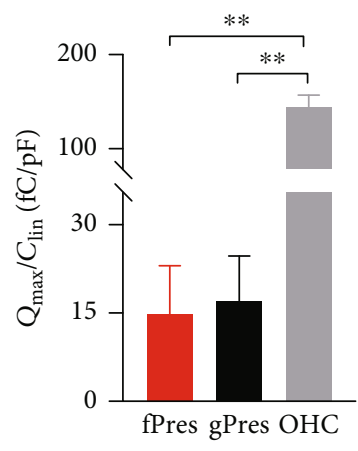

(b)

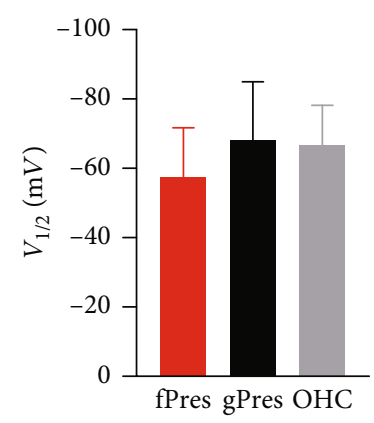

(c)

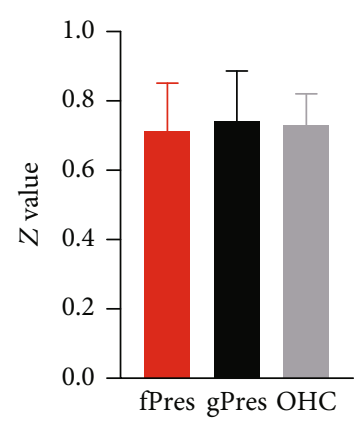

(d)

Figure 3: NLC functions of fPres, gPres, and mouse OHC. (a-d) Showed four parameters derived from curve fittings with Boltzmann's function for fPres $(n=16)$, gPres $(n=15)$, and OHC $(n=6)$. Data were expressed as mean \pm s.d. ${ }^{*} P<0.05,{ }^{* *} P<0.01$.

TABLE 1: All the measurements performed in the present study are expressed as mean \pm sem.

\begin{tabular}{lccccc}
\hline & $C_{\operatorname{lin}}(\mathrm{pF})$ & $Q_{\max }(\mathrm{fC})$ & $V_{1 / 2}(\mathrm{mV})$ & $z$ & $Q_{\max } / C_{\operatorname{lin}}(\mathrm{fC} / \mathrm{pF})$ \\
\hline $\mathrm{AP}(n=10)$ & $14.9 \pm 1.01$ & $10.4 \pm 1.4$ & $-33.8 \pm 3.3$ & $1.8 \pm 0.16$ & $0.69 \pm 0.07$ \\
$\mathrm{~S}(n=8)$ & $16.4 \pm 0.68$ & $19.9 \pm 2.4$ & $-20.7 \pm 3.3$ & $2.4 \pm 0.08$ & $1.25 \pm 0.19$ \\
fPres $(n=16)$ & $12.7 \pm 0.91$ & $181.5 \pm 22.5$ & $-58.1 \pm 3.5$ & $0.07 \pm 0.03$ & $14.9 \pm 2.02$ \\
gPres $(n=15)$ & $15.9 \pm 1.03$ & $279.4 \pm 41.7$ & $-68.3 \pm 4.4$ & $0.07 \pm 0.04$ & $16.9 \pm 2$ \\
Mouse OHC $(n=9)$ & $5.74 \pm 0.14$ & $778.6 \pm 26.2$ & $-71.5 \pm 3.6$ & $0.77 \pm 0.03$ & $136.4 \pm 5.98$ \\
\hline
\end{tabular}

the functional activity and found that the charge density, $z$ value, and $V_{1 / 2}$ of fPres were very similar to that of gPres.

The otoacoustic emissions (OAEs) revealed much about the physiology of the ear. In mammals, OAEs were considered to be the active process generated by the electromotility of the outer HC. The nonmammalian vertebrate inner ear also exhibits an active process, and it is very interesting that the overall emission levels of amphibian ears is the largest, followed by the mammals, and then birds, which have the smallest emission level [19]. In addition to the role of the $\mathrm{HC}$ bundle in the active process, we cannot rule out the effect of prestin in amphibian HCs. Since no previous studies have measured amphibian HCs in auditory organs, we did not know if they generated NLC. We used the same voltage stimulus protocol to record HCs isolated from the AP, BP, and S from Rana catesbeiana. HCs of the AP and S displayed bellshaped voltage-dependent NLC, while the cells from BP did not. Notably, our results explained the SOAE test reported by van Dijk et al., who measured SOAE in five frog species, including Rana catesbeiana. The highest emission frequency they tested was $1735 \mathrm{~Hz}$, which was within the AP frequency range, and no emissions were recorded in the BP range [17]. It is likely that the prestin expression in the BP HCs was too low to be detected. Another explanation is that the inner ear of frog functions well at a very low frequency. The BP did not act like the AP and S, or even lost its active process ability for its relatively higher frequency sensing range. The $Q_{\max }$ and charge density of $S$ were higher than that measured in the AP cells. These results may be due to the larger cell size in the $\mathrm{S}$ organ, or there might be more prestin expressed in the cell membrane of its tissue. Since charge density directly correlates with the level of prestin expression at the membrane, it is reasonable that $\mathrm{S}$ has a larger magnitude of NLC than AP [42].

When we compared the results of frog HCs and mouse OHCs, the charge density was dramatically different between these two taxa. The mouse OHC prestin had a greater charge density than the frog prestin, along with a significant shift of $V_{1 / 2}$ from positive to negative potentials. It is suggested that the functional evolution of prestin lies in the acquisition of NLC and the potential for $V_{1 / 2}$ to shift from positive to negative $[26,43]$. Our study supports the hypothesis that the amphibian prestin is evolutionarily less advanced than mammalian prestin.

As we know, there is a charged voltage sensor within prestin that moves through the electrical field and gives rise to an electric current. This electric current, similar to a gating current, generates NLC. The $z$ value was quite different between the mammalian and amphibian prestin proteins; however, we did not measure the motility or transport function of the frog prestin in this report. In previous studies, there is a reciprocal trend between NLC magnitude and anion transport properties during the functional evolution of prestin [44]. According to our results that the OHC has more prominent NLC than its nonmammalian orthologs, the transport capability of frog prestin might be stronger and its anion transport capability could be the dominant function of frog prestin. Nevertheless, without direct measurement of motility, the contribution of frog prestin to electromotility cannot be completely ruled out. 


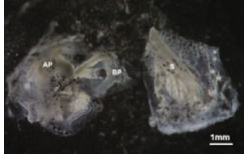

(a)

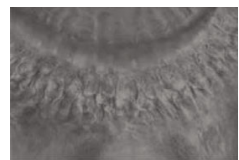

(e)

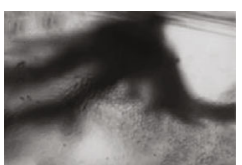

(b)

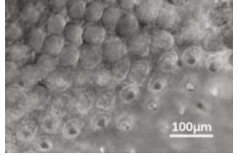

(c)

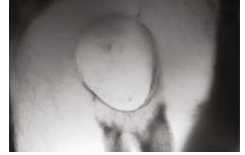

(d)

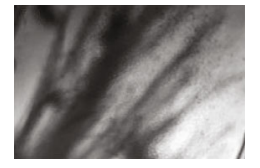

(f)

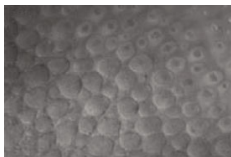

(g)

FIGURE 4: Images of frog's hearing organ. (a) Dissection of the frog's inner ear which contained three auditory organs (AP, BP, and S) under a 10x microscope. (b, c) Displayed was a higher magnification image of the AP under a 100x and 600x microscope. (d, e) Displayed was a higher magnification image of the BP under a 100x and 600x microscope. (f, g) Displayed was a higher magnification image of the $S$ under a 100x and 600x microscope.

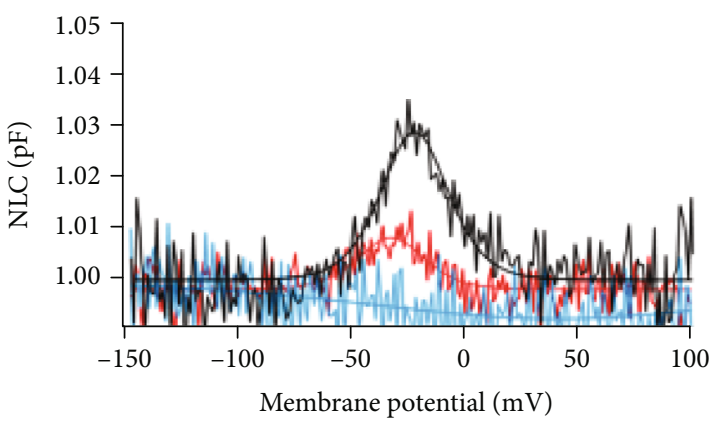

(a)
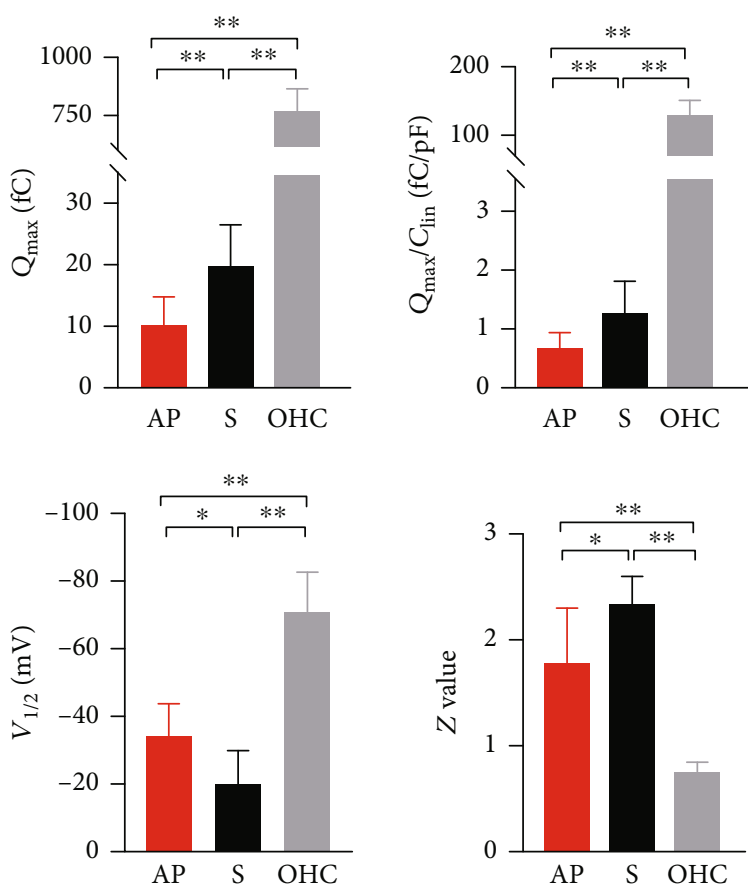

(b)

FIGURE 5: NLC functions of frog's three auditory organs (AP, BP, and S). (a) NLC obtained from the hair cells of three auditory organs. Red: the amphibian papilla (AP); black: the sacculus (S); blue: the basilar papilla (BP). No NLC was detected in the hair cells of the basilar papilla (BP). (b) Four parameters derived from curve fittings with Boltzmann's function for AP $(n=10)$ and $\mathrm{S}(n=8)$. Mouse OHC was used as a comtrol. Data are expressed as mean \pm s.d. ${ }^{* *} P<0.01,{ }^{*} P<0.05$ (Student's $t$-test).

\section{Conclusions}

We observed acquisition of NLC both in fPres-transfected cells and in HCs isolated from frog auditory organs. Our results represent the first and necessary step in revealing possible roles of prestin in the active hearing processes found in many nonmammalian species. This might lead to the alternative hypothesis that both prestin and HC bundles might function together as the intrinsic kinetics for amplification and frequency selectivity in amphibian inner ears.

\section{Data Availability}

The data (data for prestin of bullfrog) used to support the findings of this study are included within the supplementary information file(s).

\section{Conflicts of Interest}

The authors declare that they have no conflicts of interest. 


\section{Acknowledgments}

We thank Professor Geng-lin Li and professor Lei Song for technical support in electrophysiology. We also thank Geng-lin Li for the comments on our manuscript draft. This work was funded by the National Natural Science Foundation of China (NSFC) to H.W. (81730028) and the Shanghai Key Laboratory of Translational Medicine on Ear and Nose Diseases (14DZ2260300). The authors declare no competing financial interests.

\section{Supplementary Materials}

In our supplementary material, you may obtain all the NLC measurements of the mouse OHCs, HEK293T cells transfected with gPres and fPres, AP HCs, and S HCs from the frog auditory organ. (Supplementary Materials)

\section{References}

[1] Y. Liu, J. Qi, X. Chen et al., "Critical role of spectrin in hearing development and deafness," Science Advances, vol. 5, no. 4, p. eaav7803, 2019.

[2] J. Qi, Y. Liu, C. Chu et al., "A cytoskeleton structure revealed by super-resolution fluorescence imaging in inner ear hair cells," Cell Discovery, vol. 5, no. 1, p. 12, 2019.

[3] X. Lu, S. Sun, J. Qi et al., "Bmil regulates the proliferation of cochlear supporting cells via the canonical Wnt signaling pathway," Molecular Neurobiology, vol. 54, no. 2, pp. 1326-1339, 2017.

[4] B. Kachar, W. E. Brownell, R. Altschuler, and J. Fex, "Electrokinetic shape changes of cochlear outer hair cells," Nature, vol. 322, no. 6077, pp. 365-368, 1986.

[5] J. Santos-Sacchi and W. Tan, "The frequency response of outer hair cell voltage-dependent motility is limited by kinetics of prestin," The Journal of Neuroscience, vol. 38, no. 24, pp. 5495-5506, 2018.

[6] J. Santos-Sacchi and L. Song, "Chloride anions regulate kinetics but not voltage-sensor Qmax of the solute carrier SLC26a5," Biophysical Journal, vol. 110, no. 11, pp. 25512561, 2016.

[7] T. Wang, R. Chai, G. S. Kim et al., "Lgr5+ cells regenerate hair cells via proliferation and direct transdifferentiation in damaged neonatal mouse utricle," Nature Communications, vol. 6, no. 1, p. 6613, 2015.

[8] B. C. Cox, R. Chai, A. Lenoir et al., "Spontaneous hair cell regeneration in the neonatal mouse cochlea in vivo," Development, vol. 141, no. 4, pp. 816-829, 2014.

[9] R. G. Kavlie, J. L. Fritz, F. Nies et al., "Prestin is an anion transporter dispensable for mechanical feedback amplification in Drosophila hearing," Journal of Comparative Physiology. A, Neuroethology, Sensory, Neural, and Behavioral Physiology, vol. 201, no. 1, pp. 51-60, 2015.

[10] J. Santos-Sacchi and W. Tan, "Voltage does not drive prestin (SLC26a5) electro-mechanical activity at high frequencies where cochlear amplification is best," iScience, vol. 22, pp. 392-399, 2019.

[11] Y. N. Chang, E. A. Jaumann, K. Reichel et al., "Structural basis for functional interactions in dimers of SLC26 transporters," Nature Communications, vol. 10, no. 1, p. 2032, 2019.
[12] S. Takahashi, T. Yamashita, K. Homma et al., "Deletion of exons 17 and 18 in prestin's STAS domain results in loss of function," Scientific Reports, vol. 9, no. 1, p. 6874, 2019.

[13] M. F. Kuwabara, K. Wasano, S. Takahashi et al., "The extracellular loop of pendrin and prestin modulates their voltagesensing property," The Journal of Biological Chemistry, vol. 293, no. 26, pp. 9970-9980, 2018.

[14] S. Lovas, D. Z. Z. He, H. Liu et al., "Glutamate transporter homolog-based model predicts that anion- $\pi$ interaction is the mechanism for the voltage-dependent response of prestin," The Journal of Biological Chemistry, vol. 290, no. 40, pp. 24326-24339, 2015.

[15] R. Guo, S. Zhang, M. Xiao et al., "Accelerating bioelectric functional development of neural stem cells by graphene coupling: implications for neural interfacing with conductive materials," Biomaterials, vol. 106, pp. 193-204, 2016.

[16] A. Chang, C. Li, J. Huang, W. Pan, Y. Tian, and J. Tang, “Auditory brainstem response and outer hair cell whole-cell patch clamp recording in postnatal rats," Journal of Visualized Experiments, vol. 135, no. 135, 2018.

[17] P. van Dijk, P. M. Narins, and J. Wang, "Spontaneous otoacoustic emissions in seven frog species," Hearing Research, vol. 101, no. 1-2, pp. 102-112, 1996.

[18] R. Chai, B. Kuo, T. Wang et al., "Wnt signaling induces proliferation of sensory precursors in the postnatal mouse cochlea," Proceedings of the National Academy of Sciences of the United States of America, vol. 109, no. 21, pp. 8167-8172, 2012.

[19] C. Bergevin, D. M. Freeman, J. C. Saunders, and C. A. Shera, "Otoacoustic emissions in humans, birds, lizards, and frogs: evidence for multiple generation mechanisms," Journal of Comparative Physiology. A, Neuroethology, Sensory, Neural, and Behavioral Physiology, vol. 194, no. 7, pp. 665-683, 2008.

[20] L. Chen, W. Sun, and R. J. Salvi, "Electrically evoked otoacoustic emissions from the chicken ear," Hearing Research, vol. 161, no. 1-2, pp. 54-64, 2001.

[21] M. Beurg, X. Tan, and R. Fettiplace, “A prestin motor in chicken auditory hair cells: active force generation in a nonmammalian species," Neuron, vol. 79, no. 1, pp. 69-81, 2013.

[22] T. A. Jan, R. Chai, Z. N. Sayyid et al., “Tympanic border cells are Wnt-responsive and can act as progenitors for postnatal mouse cochlear cells," Development, vol. 140, no. 6, pp. 1196-1206, 2013.

[23] R. L. M. Schoffelen, J. M. Segenhout, and P. van Dijk, "Mechanics of the exceptional anuran ear," Journal of Comparative Physiology. A, Neuroethology, Sensory, Neural, and Behavioral Physiology, vol. 194, no. 5, pp. 417-428, 2008.

[24] S. Ji, D. Bozovic, and R. Bruinsma, "Amphibian sacculus and the forced Kuramoto model with intrinsic noise and frequency dispersion," Physical Review E, vol. 97, no. 4, article 042411, 2018.

[25] J. B. Azimzadeh and J. D. Salvi, "Physiological preparation of hair cells from the sacculus of the American bullfrog (Rana catesbeiana)," Journal of Visualized Experiments, vol. 121, no. 121, 2017.

[26] X. Tan, J. L. Pecka, J. Tang, S. Lovas, K. W. Beisel, and D. Z. Z. $\mathrm{He}$, "A motif of eleven amino acids is a structural adaptation that facilitates motor capability of eutherian prestin," Journal of Cell Science, vol. 125, no. 4, pp. 1039-1047, 2012. 
[27] W. Chen, L. Tan, Q. Zhou et al., "AAVS1 site-specific integration of the CAR gene into human primary T cells using a linear closed-ended AAV-based DNA vector," The Journal of Gene Medicine, vol. 22, no. 4, p. e3157, 2020.

[28] H. Yang, J. Wang, M. Zhao et al., "Feasible development of stable HEK293 clones by CRISPR/Cas9-mediated site-specific integration for biopharmaceuticals production," Biotechnology Letters, vol. 41, no. 8-9, pp. 941-950, 2019.

[29] J. Zhang, X. Liu, Y. Zhang et al., "Human neural stem cells with GDNF site-specific integration at AAVS1 by using AAV vectors retained their stemness," Neurochemical Research, vol. 43, no. 4, pp. 930-937, 2018.

[30] F. Tan, C. Chu, J. Qi et al., "AAV-ie enables safe and efficient gene transfer to inner ear cells," Nature Communications, vol. 10, no. 1, p. 3733, 2019.

[31] Y. Luo, A. Frederick, J. M. Martin et al., "AAVS1-targeted plasmid integration in AAV producer cell lines," Human Gene Therapy Methods, vol. 28, no. 3, pp. 124-138, 2017.

[32] C. Li, A. S. Mishra, S. Gil et al., "Targeted integration and highlevel transgene expression in AAVS1 transgenic mice after in vivo HSC transduction with HDAd5/35++ vectors," Molecular Therapy, vol. 27, no. 12, pp. 2195-2212, 2019.

[33] R. Fettiplace, "Diverse mechanisms of sound frequency discrimination in the vertebrate cochlea," Trends in Neurosciences, vol. 43, no. 2, pp. 88-102, 2020.

[34] E. Lewis, R. Baird, E. Leverenz, and H. Koyama, "Inner ear: dye injection reveals peripheral origins of specific sensitivities," Science, vol. 215, no. 4540, pp. 1641-1643, 1982.

[35] Z. H. He, S. Y. Zou, M. Li et al., "The nuclear transcription factor FoxG1 affects the sensitivity of mimetic aging hair cells to inflammation by regulating autophagy pathways," Redox Biology, vol. 28, p. 101364, 2020.

[36] W. Liu, X. Xu, Z. Fan et al., "Wnt signaling activates TP53induced glycolysis and apoptosis regulator and protects against cisplatin-induced spiral ganglion neuron damage in the mouse cochlea," Antioxidants \& Redox Signaling, vol. 30, no. 11, pp. 1389-1410, 2019.

[37] Z. He, L. Guo, Y. Shu et al., "Autophagy protects auditory hair cells against neomycin-induced damage," Autophagy, vol. 13, no. 11, pp. 1884-1904, 2017.

[38] L. Liu, Y. Chen, J. Qi et al., "Wnt activation protects against neomycin-induced hair cell damage in the mouse cochlea," Cell Death \& Disease, vol. 7, no. 3, p. e2136, 2016.

[39] Z. He, Q. Fang, H. Li et al., "The role of FOXG1 in the postnatal development and survival of mouse cochlear hair cells," Neuropharmacology, vol. 144, pp. 43-57, 2019.

[40] S. Zhang, Y. Zhang, Y. Dong et al., "Knockdown of Foxg1 in supporting cells increases the trans-differentiation of supporting cells into hair cells in the neonatal mouse cochlea," Cellular and Molecular Life Sciences, vol. 77, no. 7, pp. 1401-1419, 2020.

[41] W. Yan, W. Liu, J. Qi et al., "A three-dimensional culture system with Matrigel promotes purified spiral ganglion neuron survival and function in vitro," Molecular Neurobiology, vol. 55, no. 3, pp. 2070-2084, 2018.

[42] M. L. Seymour, L. Rajagopalan, G. Duret et al., "Membrane prestin expression correlates with the magnitude of prestinassociated charge movement," Hearing Research, vol. 339, pp. 50-59, 2016.
[43] J. Tang, J. L. Pecka, B. Fritzsch, K. W. Beisel, and D. Z. Z. He, "Lizard and frog prestin: evolutionary insight into functional changes," PLoS One, vol. 8, no. 1, article e54388, 2013.

[44] X. Tan, J. L. Pecka, J. Tang et al., "From zebrafish to mammal: functional evolution of prestin, the motor protein of cochlear outer hair cells," Journal of Neurophysiology, vol. 105, no. 1, pp. 36-44, 2011. 